\title{
COMPACTIFICATION AND DUALITY OF TOPOLOGICAL GROUPS
}

\author{
BY \\ HSIN CHU(1)
}

1. Introduction. In this paper we give a unified approach to two distinct theorics in topological groups. On the one hand we have the Pontrjagin and Tannaka dualities for locally compact abelian groups and compact groups, respectively. On the other hand, in abstract harmonic analysis, we have the compactification of a group in the sense of Weil (see [2], [3], [13], [19]). Let $G$ be a topological group. Let $G_{d}^{x}$ and $G^{x}$ be the set of all unitary representations (finite dimensional) with the discrete topology and with the compact open topology, respectively. Let $\left(G_{d}^{x}\right)^{x}$ and $G^{x x}$ be the groups with the compact open topology of all unitary mappings (see Definition 2) of $G_{d}^{x}$ and $G^{x}$ respectively. These groups are always maximally almost periodic in the sense of J. von Neumann. Let $\tilde{G}$ be the compact group attached to $G$ (i.e. $\tilde{G}$ is the compactification of $G$ in the sense of Weil). We prove that for any topological group, $\left(G_{d}^{x}\right)^{x} \cong \widetilde{G}$, which gives rise to a new method for constructing the compact group $\tilde{G}$. In particular, if $G$ is abelian, then $\left(G_{d}^{*}\right)^{*}=\tilde{G}$, where $G_{d}^{*}$ is the character group of $G$ with the discrete topology and $\left(G_{d}^{*}\right)^{*}$ is the character group of $G_{d}^{*}$ with the compact open topology, which is an extension of a known result of Kakutani (see [2] and [3]). Let $G \cong A \times B$ be the direct product of two topological groups. We prove that $\tilde{G} \cong \tilde{A} \times \widetilde{B}$ and $G^{x x} \cong A^{x x} \times B^{x x}$. A similar result is known for Stone-Čech compactification of certain spaces. If $G$ is locally compact, then $\tilde{G}^{x x} \cong \tilde{G}$. If $G$ is maximally almost periodic, locally compact with a $\sigma$-compact commutator and it has a compact normal subgroup $A$ such that $(G / A)^{x x} \cong G / A$ in a natural sense, then $G^{x x} \cong G$, which is a generalization of Pontrjagin duality and Tannaka duality. In particular, if $G$ is maximally almost periodic, locally compact with a compact commutator, then $G^{x x} \cong G$. In [17], Takahashi proved the same statement except he used a topology for $G^{x}$ other than the usual compact-open topology. In other directions, there are several papers which recently studied Tannaka duality alone; for example, see [1], [5], [9], [10], [11], [16]. Throughout the paper, the Pontrjagin duality and the Tannaka duality are assumed.

Presented to the Society, February 23, 1963; received by the editors April 30, 1963.

(1) This work was partially supported by Contract NAS8-1646 with the George C. Marshall Space Flight Center, Huntsville, Alabama. 


\section{Topologies for $G^{x}$ and $G^{x x}$.}

Definition 1. Let $G$ be a topological group. Let $G_{n}^{x}$ be the set of all unitary representations of degree $n$ of $G$ with the usual compact open topology. Let $G^{x}=\bigcup_{n} G_{n}^{x}$ as the sum of all topological sets $G_{n}^{x}, n=1,2, \cdots$. We call $G^{x}$ the unitary space of $G$ and $G_{n}^{x}$ the unitary subspace of degree $n$ of $G$. We know that $G^{x}$ is closed with respect to the following operations: $\operatorname{sum}(\dot{+})$, Kronecker product $(X)$, equivalence, and complex conjugate. We denote

$$
\begin{aligned}
U(D, K, \varepsilon)= & U(D)=\left\{D^{\prime} \mid\left\|D(g)-D^{\prime}(g)\right\|<\varepsilon,\right. \text { where } \\
& g \in K \subset G, K \text { is a compact set in } G, D \\
& \text { and } \left.D^{\prime} \text { in } G_{n}^{x}, \text { and } \varepsilon \text { is a positive real number }\right\}
\end{aligned}
$$

as a ncighborhood of $D$, where $\left\|D(g)-D^{\prime}(g)\right\|=\left(\Sigma_{i j}\left(d_{i j}(g)-d_{i j}^{\prime}(g)\right)\right)^{1 / 2}$.

LEMMA 1. The set of all neighborhoods $U(D, K, \varepsilon)$ forms a base of a topology for $G_{n}^{x}$. Moreover, it is a Hausdorff uniform space.

Proof. It is a straighforward computation.

LEMMA 2. The subspace $G_{n}^{x}$ is locally compact for each $n$, if $G$ is locally compact.

Proof. This lemma is known. For example, it is a direct consequence of Theorem 1 in [8].

Corollary. The dual space $G=\bigcup_{n} G_{n}^{x}$ is a locally compact Hausdorff uniform space, if $G$ is locally compact.

Definition 2. A unitary mapping $A$ of $G$ is a continuous function of $G^{x}$ into the set of all unitary groups $U(n), n=1,2, \cdots$, such that:

(I) $A(D) \in U(d(D))$,

(II) $A\left(D+D^{\prime}\right)=A(D)+A\left(D^{\prime}\right)$,

(III) $A\left(D \times D^{\prime}\right)=A(D) \times A\left(D^{\prime}\right)$,

(IV) $A\left(T^{-1} D T\right)=T^{-1} A(D) T$,

where $D, D^{\prime} \in G, d(D)=$ the degree of $D$ and $T \in U(d(D))$.

If $A$ is a unitary mapping of $G$, then it is known that $(A(D))^{-}=A(\bar{D})$. (See [18].) Denote the set of all unitary mappings of $G^{x}$ by $G^{x x}$. Let us introduce a multiplication of $A$ and $B$ in $G^{x x}$ by $(A B)(D)=A(D) B(D)$ for $D \in G^{x}$. Define $A^{-1}$ for $A \in G^{x x}$ by $A^{-1}(D)=(A(D))^{-1}$ for all $D \in G$. Introduce the usual compact open topology to $G^{x x}$. Then it is easy to verify the following lemma.

Lemma 3. The set $G^{x x}$ is a topological (Hausdorff) group and $G^{x x}$ is also maximally almost periodic in the sense of John von Neumann. (See [14].)

LEMMA 4. If $G$ is a discrete group, then $G_{n}^{x}$ is a compact space and therefore $G^{x}$ is $\sigma$-compact. 
Proof. Let $U(n)$ be the group of all unitary representations of degree $n$ over the complex field $C$. Then the product group $\prod_{g \in G} U_{g}(n)$ is compact, where $U_{g}(n) \cong U(n)$ for every $g \in G$.

Define

$$
f: G_{n}^{x} \rightarrow \prod_{g \in G} U_{g}(n) \text { by } f(D)=\{D(g)\} \quad \text { for all } g \in G, D \in G_{n}^{x} .
$$

It is not hard to see that $f$ is a homeomorphic into mapping and $f\left(G_{n}^{x}\right)$ is closed in $\prod_{g \in G} U_{g}(n)$. This proves that $G_{n}^{x}$ is compact.

LEMMA 5. Let $G$ be a locally compact group satisfying the second axiom of countability. Then $G_{n}^{x}$ satisfies the second axiom of countability and so does $G^{x}$.

Proof. We may imitate a method used in $[15, \mathrm{pp} .128-130]$ for the proof of this lemma.

Corollary 1. Let $G$ be a locally compact group satisfying the second axiom of countability. Then $G^{x}$ is metrizable.

Let $U$ and $V$ be two equivalent unitary representations of the same degree $n$ of a topological group $G$, i.e., there exists a constant matrix $P \in G L(n, C)$ such that $P^{-1} U(x) P=V(x)$ for $x \in G$. Then it is known that $P=Q \cdot a \cdot 1_{n}$ where $Q$ is a unitary matrix and $a$ is a positive real number, that is, two unitary representations are equivalent if and only if they are unitarily equivalent. We denote $U R V$ for two equivalent unitary representations $U$ and $V$. It is clear that $R$ is an equivalence relation.

LeMmA 6. Let $G$ be a compact group. Then there exists for each $C^{x} \in G_{n}^{x}$ a neighborhood $V=V\left(C^{x} ; G, 1 / n\right)$ such that all elements in $V$ are unitarily equivalent to $C^{x}$. Consequently, the quotient space $G_{n}^{x} / R$ is discrete.

Proof. Let $d x$ be the Haar measure of $G$ normalized by $\int_{g} d x=1$. Let $x_{i}$ be irreducible characters and $a_{i}$ be integers, $\mathrm{i}=1, \cdots, m$. Let $x=\boldsymbol{\Sigma} a_{i} x_{i}$. Then, by the orthogonality relations, we have $\int x \bar{x} d x=\Sigma a_{i}^{2} \geqq 1$, unless all $a_{i}, i=1, \cdots, m$, are zero. By using this fact, it is easy to see that $V\left(C^{x}, G, 1 / n\right)$ contains no elements other than those unitarily equivalent to $C^{x}$.

The following theorem, which is a direct consequence of Lemma 4 and Lemma 6, is analogous to a known theorem of locally compact abelian groups:

THEOREM 1. If $G$ is a discrete group, then $G_{n}^{x}$ is compact. If $G$ is a compact group, then $G_{n}^{x}$, modulo the unitary equivalence relation, is discrete.

LEMMA 7. Let $G$ be a locally compact group. If $H$ is a closed normal subgroup of $G$, then $\left(G_{n}^{x}, H\right)$ is a closed subset of $G_{n}^{x}$ and $(G / H)_{n}^{x}$ is topologically homeomorphic to $\left(G_{n}^{x}, H\right)$. Consequently, $(G / H)^{x} \approx\left(G^{x}, H\right)$, where 
and

$$
\left(G_{n}^{x}, H\right)=\left\{D \mid D \in G_{n}^{x}, D(h)=1_{n} \text { for all } h \in H\right\}
$$

$$
\left(G^{x}, H\right)=\left\{D \mid D \in G^{x}, D(h)=1_{d(D)} \text { for all } h \in H\right\} .
$$

Proof. For each $D \in\left(G_{n}^{x}, H\right)$, define $D^{\prime}(x H)=D(x)$ for each $x \in G$. It is clear that $D^{\prime} \in(G / H)_{n}^{x}$. Define $f:\left(G_{n}^{x}, H\right) \rightarrow(G / H)_{n}^{x}$ by $f(D)=D^{\prime}$ where $D \in\left(G_{n}^{x}, H\right)$. It is not hard to see that $f$ is one-to-one, onto and open mapping. We shall show that $f$ is continuous.

Let $V^{\prime}=V^{\prime}\left(D_{n}^{\prime} ; F H, \varepsilon\right)$ be a neighborhood of $D_{n}^{\prime}$ in $(G / H)_{n}^{x}$, where $F H$ is compact in $G / H$. For each $x \in F$ and a neighborhood $V$ of identity in $G$ whose closure, $\bar{V}$, is compact, we have $F H \subset \bigcup_{x \in F} x V H$. Because $F H$ is compact in $G / H$, there exists a finite set $F^{\prime}=\left\{x_{1}, \cdots, x_{n}\right\} \subset F$ such that $F H \bigcup_{i=1}^{n} x_{i} V H$ and $F^{\prime} \bar{V}$ is compact in $G$. Define $V^{\prime \prime}=V^{\prime \prime}\left(D_{n}^{\prime} ; F^{\prime} \bar{V} H, \varepsilon\right)$ to be a neighborhood of $D_{n}^{\prime}$ in $(G / H)_{n}^{x}$. It is easy to see that $V^{\prime \prime} \subset V^{\prime}$. Define $V=V\left(D_{n} ; F^{\prime} \bar{V}, \varepsilon\right)$ a neighborhood of $D_{n}$ in $\left(G_{n}^{x}, H\right)$, where $f\left(D_{n}\right)=D_{n}^{\prime}$. Then $f(V)=V^{\prime \prime} \subset V^{\prime}$. This proves that $f$ is continuous. Hence, $\left(G_{n}^{x}, H\right) \approx(G / H)_{n}^{x}$. Consequently, $\left(G^{x}, H\right) \approx(G / H)^{x}$.

The following theorem is analogous to a known theorem of locally compact abelian groups.

THeORem 2. Let $G$ be a locally compact, maximally almost periodic group. Let $H$ be a closed normal subgroup of $G$. Let $\Phi=\left(G^{x}, H\right)=\left\{g^{x} \mid g^{x} \in G^{x}\right.$ and $g^{x}(h)=1_{n}$ for all $h \in H$, where $n=$ degree of $\left.g^{x}\right\}$ and let $H^{\prime}=(G, \Phi)=\{g \mid \zeta(g)$ $=1_{n}$ for all $\zeta \in \Phi$ where $n=$ degree of $\left.\zeta\right\}$. If $G / H$ is maximally almost periodic then

$$
H^{\prime}=H \text { or } H=\left(G,\left(G^{x}, H\right)\right) .
$$

Proof. We know that

$$
H^{\prime}=\bigcap_{\zeta \in \Phi}\left\{g \mid \zeta(g)=1_{n}, \text { where } n=\text { degree of } \zeta\right\} .
$$

This shows that $H^{\prime}$ is a closed normal subgroup of $G$. It is easy to see, by the definition of $H^{\prime}$, that $H \subset H^{\prime}$. We shall show that $H^{\prime} \subset H$. Suppose there is an $a \in H^{\prime} \mid H$. Then $a H \neq H$ and $\zeta(x)=1_{d(\zeta)}$ for all $\zeta \in \Phi$, where $d(\zeta)$ is the degree of $\zeta$. Consequently, $\zeta(a h)=1_{d(\zeta)}$, for all $\zeta \in \Phi$ and all $h \in H$. By Lemma 7, we know $f: \Phi=\left(G^{x}, H\right) \rightarrow(G / H)^{x}$ by $f(\zeta)=\zeta^{\prime}$ where $\zeta \in H$ and $\zeta^{\prime}(x H)=\zeta(x)$ for $x H \in G / H$ is a homeomorphic onto mapping. Then $f(\zeta)(a H)=1_{d(\zeta)}$ for all $\zeta \in H$. By the hypothesis, $G / H$ is maximally almost periodic. For $a H \in G / H$ which is not identity, we have, by definition, $f\left(\zeta_{1}\right) \in(G / H)^{x}$, for some $\zeta_{1} \in \Phi$ such that $\left[f\left(\zeta_{1}\right)\right](a H) \neq 1_{d\left(\zeta_{1}\right)}$. This gives a contradiction to our earlier conclusion. Hence, $H^{\prime}=H$.

The following lemma is clear.

LEMMA 8. Let $G$ be a topological group. Let $g \in G$ and define $\hat{g}(D)=D(g)$ for all $D \in G^{x}$. Then $\hat{g} \in G^{x x}$. Define $\phi: G \rightarrow G^{x x}$ by $\phi(g)=\hat{g}$. Then $\phi$ is a contin- 
uously homomorphic mapping of $G$ into $G^{x x}$ if $G$ is locally compact. Moreover, if $G$ is maximally almost periodic, then $\phi$ is isomorphic.

Definition 3. Let $G$ be a topological group and let $G$ and $G^{x x}$ and a mapping $\phi$ as defined in the Lemma 8 . If $\phi$ is a topologically isomorphic onto mapping, then we say that $G$ has a unitary duality.

For example, locally compact abelian groups, compact groups, free groups with finite generators with discrete topology, etc., are maximally almost periodic.

3. Unitary duality and Tannaka duality. Let $G$ be a compact group and let $G^{\star}$ be the set of all representations of $G$ with the discrete topology. Define a representation mapping $A$ of $G^{\star}$ to be a mapping of $G$ into the set of $\bigcup_{m=1}^{\infty} G L(n, C)$ such that (I) $A(D) \in G L(d(D), C)$, (II) $A\left(D+D^{\prime}\right)=A(D)+A\left(D^{\prime}\right)$, (III) $A\left(D \times D^{\prime}\right)$ $=A(D) \times A\left(D^{\prime}\right)$, (IV) $(A(D))^{-}=A(\bar{D})$, and (V) $A\left(P^{-1} D P\right)=P^{-1} A(D) P$, where $D$ and $D^{\prime}$ in $G^{\star}, P \in G L(d(D), C)$. Denote the set of all representation mappings with the usual finite open topology by $G^{\star \star}$. Introduce the same multiplication in $G^{\star \star}$ as in $G^{x x}$. Define $\phi^{\prime}: G \rightarrow G^{\star \star}$ in the same way as defined in Lemma 8. It is well known that $\phi^{\prime}$ is a topological isomorphic onto mapping, and it is called the Tannaka duality (see [18]).

THEOREM 3. Let $G$ be a compact group. Then $G$ has the unitary duality which is the same as the Tannaka duality.

Proof. The proof of this theorem is a matter of straightforward computation.

COROLlary 1. Let $G$ be a compact group. Let $G_{d}^{x}$ be the set $G^{x}$ with the usual discrete topology. Let $\left(G_{d}^{x}\right)^{x}$ be the set of all unitary mappings (see Definition 2) defined on $G_{d}^{x}$ with the finite open topology. Introduce a natural multiplication in $\left(G_{d}^{x}\right)^{x}$ as we did for $G^{x x}$. Define $\phi: G \rightarrow\left(G_{d}^{x}\right)^{x}$ as we defined in the Definition 3. Then $\phi$ is a topologically isomorphic onto mapping from the topological group $G$ onto the topological group $\left(G_{d}^{x}\right)^{x}$.

4. Unitary duality and Pontrjagin duality. Let $G_{1}^{x}=\{$ the set of all unitary representations of degree one of a locally compact abelian group $G\}$. We use the Kronecker product of $G^{x}$ as the multiplication for $G_{1}^{x}$. Then $G_{1}^{x}$ is a topological group such that $G_{1}^{x} \cong G^{*}$, where $G^{*}$ is the character group of $G$ with the compact open topology. Let $G_{1}^{x x}=\{$ the set of all one-dimensional unitary representations of the topological group $\left.G_{1}^{x}\right\}$, with the compact open topology. Then $G_{1}^{x x} \cong G^{* *}$, where $G^{* *}$ is the character group of $G^{*}$ with the compact open topology.

THEOREM 4. Let $G$ be a locally compact abelian group, then $G$ has the unitary duality which is the same as the Pontrjagin duality.

Proof. The proof of this theorem is a matter of straightforward computation. 
5. Unitary duality on direct product groups. The following fact is known (e.g. see [19]).

LEMma 9. Let $G=A \times B$ be a direct product of two closed normal groups $A$ and $B$. Let $D$ be an irreducible unitary representation of the topological group $G$. Then there exist two irreducible unitary representations $D_{(1)}$ and $D_{(2)}$ of $A$ and $B$ respectively such that $D$ is unitarily equivalent to $D_{(1)}^{\prime} \times D_{(2)}^{\prime}$, where $D_{(1)}^{\prime}(a)=D_{(1)}(a)$ and $D_{(1)}^{\prime}(b)=1_{d\left(D_{(1)}\right)}$ and $D_{(2)}^{\prime}(b)=D_{(2)}(b)$ and $D_{(2)}^{\prime}(a)$ $=1_{a\left(D_{(2)}\right)}$, where $a \in A$ and $b \in B$.

Definition 4. Let $A_{n}^{x}, B_{n}^{x}, C_{n}^{x}, D_{n}^{x}$, etc., be the unitary spaces of degree $n$ of topological groups $A, B, C, D$, etc., respectively. Let

$$
f_{n}: A_{n}^{x} \rightarrow B_{n}^{x}, g_{n}: B_{n}^{x} \rightarrow C_{n}^{x}, h_{n}: C_{n}^{x} \rightarrow D_{n}^{x} \text {, etc. }
$$

be continuous mappings such that $g_{n}^{-1}\left(1_{n}\right)=f_{n}\left(A_{n}\right), h_{n}^{-1}\left(1_{n}\right)=g_{n}\left(B_{n}\right)$, etc., where $1_{n}$ is the trivial representation of each one of $A_{n}, B_{n}, C_{n}, D_{n}$, etc. Then we call the sequence

$$
\cdots \rightarrow A_{n}^{x} \stackrel{f_{n}}{\rightarrow} B_{n}^{x} \stackrel{g_{n}}{\rightarrow} C_{n}^{x} \stackrel{h_{n}}{\rightarrow} D_{n}^{x} \rightarrow \cdots
$$

exact.

Lemma 10. Let $G$ be a locally compact group. Let $G=A \times B$ where $G$ is a direct product of two closed normal subgroups $A$ and $B$. Then

$$
1 \rightarrow A \stackrel{i}{\rightarrow} G \stackrel{j}{\rightarrow} B \rightarrow 1
$$

and

$$
1 \leftarrow A_{n}^{x} \stackrel{i_{n}^{x}}{\leftarrow} G_{n}^{x} \stackrel{j_{n}^{x}}{\leftarrow} B_{n}^{x} \leftarrow 1, \text { for all } n
$$

are exact sequences where $i$ is the inclusion mapping and $j$ is the projection and $\left[i_{n}^{x}\left(g_{n}^{x}\right)\right](a)=g_{n}^{x}(i(a)), a \in A, g_{n}^{x} \in G_{n}$ and $\left[\left(j_{n}^{x}\right)\left(b_{n}^{x}\right)\right](g)=b_{n}^{x}(j(g)), g \in G, b_{n}^{x} \in B_{n}^{x}$. Furthermore,

$$
1 \leftarrow A^{x} \stackrel{i^{x}}{\leftarrow G^{x}} \stackrel{j}{\leftarrow} B^{x} \leftarrow 1,
$$

where $i^{x}=\bigcup_{n} i_{n}^{x}$ and $j^{x}=\bigcup_{n} j_{n}^{x}$, is exact.

This lemma is a direct consequence of Lemma 7 and Lemma 9.

THEOREM 5. Let $G$ be a direct product of two topological groups $A$ and $B, G=A \times B$. Then $G^{x x} \cong A^{x x} \times B^{x x}$ is a direct product of $A^{x x}$ and $B^{x x}$.

Proof. Let

and

$$
\left(A^{x}\right)_{n}^{\prime}=\left\{g_{n}^{x} \mid g_{n}^{x} \in G_{n}^{x}, g_{n}^{x}(B)=1_{n}\right\}
$$

$$
\left(B^{x}\right)_{n}^{\prime}=\left\{g_{n}^{x} \mid g_{n}^{x} \in G_{n}^{x}, g_{n}^{x}(A)=1_{n}\right\}
$$


Let

$$
\left(A^{x}\right)^{\prime}=\bigcup_{n}\left(A^{x}\right)_{n}^{\prime} \text { and }\left(B^{x}\right)^{\prime}=\bigcup_{n}\left(B^{x}\right)_{n}^{\prime} .
$$

Define $f_{1, n}: A_{n}^{x} \rightarrow\left(A^{x}\right)_{n}^{\prime}$ by $f_{1, n}\left(a^{x}\right)(x y)=a^{x}(x)$ for $x \in A$ and $y \in B$. Define $f_{2, n}: B_{n}^{x} \rightarrow\left(B^{x}\right)_{n}^{\prime}$ by $f_{1, n}\left(b^{x}\right)(x y)=b^{x}(y)$ for $x \in A$ and $y \in B$. Define $f_{1}: A^{x} \rightarrow\left(A^{x}\right)^{\prime}$ by $f_{1}=\bigcup_{n} f_{1, n}$ and $f_{2}: B^{x} \rightarrow\left(B^{x}\right)^{\prime}$ by $f_{2}=\bigcup_{n} f_{2, n}$.

For $a^{x x} \in A^{x x}$, define $\left(a^{x x}\right)^{\prime}\left(g^{x}\right)=a^{x x}\left(g^{x} \mid A\right)$ for $g^{x} \in G^{x}$ and for $b^{x x} \in B^{x x}$, define $\left(b^{x x}\right)^{\prime}\left(g^{x}\right)=b^{x x}\left(g^{x} \mid B\right)$ for $g^{x} \in G^{x}$. Then $\left(a^{x x}\right)^{\prime}$ and $\left(b^{x x}\right)^{\prime}$ are in $G^{x x}$. Let $\left(A^{x x}\right)^{\prime}$ be the set of all these $\left(a^{x x}\right)^{\prime}$ and let $\left(B^{x x}\right)^{\prime}$ be the set of all these $\left(b^{x x}\right)^{\prime}$. We shall show that $\left(A^{x x}\right)^{\prime}$ and $\left(B^{x x}\right)^{\prime}$ are closed normal subgroups of $G^{x x}$. It is clear that $\left(A^{x x}\right)^{\prime}$ and $\left(B^{x x}\right)^{\prime}$ are subgroups of $G^{x x}$. By the definition of $\left(A^{x x}\right)^{\prime}$, we know $\left(a^{x x}\right)^{\prime}\left(b^{x}\right)^{\prime}=1_{d\left(b^{x}\right)^{\prime}}$ for each $\left(a^{x x}\right)^{\prime} \in\left(A^{x x}\right)^{\prime}$ and $\left(b^{x}\right)^{\prime} \in\left(B^{x}\right)^{\prime}$. Let $S^{x x} \in G^{x x} \backslash\left(A^{x x}\right)^{\prime}$. Then there exists $\beta^{x} \in\left(B^{x}\right)^{\prime}$ such that $g^{x x}\left(\beta^{x}\right) \neq 1_{d\left(\beta^{x}\right)}$. Suppose not, then for each $\beta^{x} \in G^{x}$, by Lemma 9 there exists $D_{1,1}, \cdots, D_{1, r}$ in $\left(A^{x}\right)^{\prime}$ and $D_{2,1}, \cdots, D_{2, r}$ in $\left(B^{x}\right)^{\prime}$ such that $\beta^{x}=S^{-1}\left(D_{1,1} \times D_{2,1}+\cdots+D_{1, r} \times D_{2, r}\right) S$ where $S \in U\left(d\left(\beta^{x}\right)\right)$ and $g^{x x}\left(\beta^{x}\right)$ $\left.=S^{-1}\left(g^{x x}\left(D_{1,1}\right)\right) \times 1_{d\left(D_{2}, 1\right)}+\cdots+g^{x x}\left(D_{2, r}\right) \times 1_{d\left(D_{2}, 1\right.}\right) S=g^{x x}\left(\beta^{x} / A\right)$.

It follows that $g^{x x} \in\left(A^{x x}\right)^{\prime}$. This contradicts the assumption $g^{x x} \in G^{x x} \mid\left(A^{x x}\right)^{\prime}$. Therefore, there is an $\beta^{x} \varepsilon\left(B^{x}\right)^{\prime}$ such that $g^{x x}\left(\beta^{x}\right) \neq 1_{d\left(\beta^{x}\right)}$. Let $\left\|g^{x x}\left(\beta^{x}\right)-1_{d\left(\beta^{x}\right)}\right\|=\eta$ and choose $\varepsilon=\eta / 2$. Then it is easy see that $W\left(g^{x x} ; \beta, \varepsilon\right) \cap\left(A^{x x}\right)^{\prime}=\varnothing$. This shows that $\left(A^{x x}\right)^{\prime}$ is closed. For each $g^{x x} \in G^{x x}$ and each $\left(a^{x x}\right)^{\prime} \in\left(A^{x x}\right)^{\prime}$, we shall show that $\left(g^{x x}\right)^{-1}\left(a^{x x}\right)^{\prime}\left(g^{x x}\right) \in A^{x x}$. For each $\gamma^{x} \in G^{x}$, by Lemma 9 , we may write $\gamma^{x}=S^{-1}\left(D_{1,1} \times D_{2,1}+\cdots+D_{1, r} \times D_{2, r}\right) S$ for $S \in U\left(d\left(\gamma^{x}\right)\right), D_{1,1}, \cdots, D_{1,2}$ in $\left(A^{x}\right)^{\prime}$ and $D_{2,1}, \cdots, D_{2, r}$ in $\left(B^{x}\right)^{\prime}$. We have

$$
\begin{aligned}
\left(g^{x x}\right)^{-1}\left(a^{x x}\right)^{\prime}\left(g^{x x}\right)\left(\gamma^{x}\right)= & \left(g^{x x}\right)^{-1}\left(a^{x x}\right)^{\prime}\left(g^{x x}\right) \\
& \cdot\left(S^{-1}\left(D_{1,1} \times D_{2,1}+\cdots+\cdots+D_{1, r} \times D_{2, r}\right) S\right) \\
= & S^{-1}\left(\left(g^{x x}\left(D_{1,1}\right)\right)^{-1}\left(\left(a^{x x}\right)^{\prime}\left(D_{1,1}\right)\right) g^{x x}\left(D_{1,1}\right) \times 1_{d\left(D_{2,1}\right)}\right) \\
& \left.+\cdots+\left(g^{x x}\left(D_{1, r}\right)\right)^{-1}\left(\left(a^{x x}\right)^{\prime}\left(D_{1, r}\right)\right) g_{x x}\left(D_{1, r}\right) \times 1_{d\left(D_{2}, r\right.}\right) S \\
= & \left(g^{x x}\right)^{-1}\left(a^{x x}\right)^{\prime}\left(g^{x x}\right)\left(\gamma^{x x} \mid A\right) .
\end{aligned}
$$

Hence $\left(g^{x x}\right)^{-1}\left(a^{x x}\right)^{\prime} g^{x x} \in\left(A^{x x}\right)^{\prime}$. This shows that $\left(A^{x x}\right)^{\prime}$ is a closed normal subgroup of $G^{x x}$. Similarly, we can show that $\left(B^{x x}\right)^{\prime}$ is a closed normal subgroup of $G^{x x}$.

For $g^{x x} \in G$, we define $g_{A}^{x x}$ and $g_{B}^{x x}$, by $g_{A}^{x x}\left(\gamma^{x}\right)=g^{x x}\left(f_{1}\left(\gamma^{x} \mid A\right)\right)$ and $g_{B}^{x x}\left(\gamma^{x}\right)$ $=g^{x x}\left(f_{2}\left(\gamma^{x} \mid B\right)\right)$ respectively. Then $g_{A}^{x x}, g_{B}^{x x} \in G^{x x}$. We shall show that $g^{x x}=g_{A}^{x x} \cdot g_{B}^{x x}$.

For each $\gamma^{x} \in G^{x}$, there exist, by Lemma $9, D_{1,1}, \cdots, D_{1, r}$ in $\left(A^{x}\right)^{\prime}$ and $D_{2,1}, \cdots, D_{2, r}$ in $\left(B^{x}\right)^{\prime}$ such that $\gamma^{x}=S^{-1}\left(D_{1,1} \times D_{1,2}+\cdots+D_{1, r} \times D_{2, r}\right) S$ where $S \in U\left(d\left(\gamma^{x}\right)\right)$.

We have

$$
\begin{aligned}
g_{A}^{x x} \cdot g_{B}^{x x}\left(\gamma^{x}\right) & =g_{A}^{x x}\left(\gamma^{x}\right) g_{B}^{x x}\left(\gamma^{x}\right) \\
& =S^{-1}\left(g^{x x}\left(D_{1,1}\right) \times g^{x x}\left(D_{2,1}\right)+\cdots+g^{x x}\left(D_{2, r}\right) \times g^{x x}\left(D_{2, r}\right)\right) S=g^{x x}\left(\gamma^{x}\right)
\end{aligned}
$$


This shows $g_{A}^{x x} \cdot g_{B}^{x x}=g^{x x}$.

We shall show $g_{A}^{x x} \in\left(A^{x x}\right)^{\prime}$ and $g_{B}^{x x} \in\left(B^{x x}\right)^{\prime}$. Define $a^{x x}\left(\alpha^{x}\right)=g_{A}^{x x}\left(f_{1}\left(\alpha^{x}\right)\right)$ for $\alpha^{x} \in A^{x}$, then $a^{x x} \in A^{x x}$ and $\left(a^{x x}\right)^{\prime}\left(\beta^{x}\right)=\left(a^{x x}\right)\left(\beta^{x} \mid A\right)$ for $\beta^{x} \in G$. Then $\left(a^{x x}\right)^{\prime}\left(\beta^{x}\right)$ $=\left(a^{x x}\right)\left(\beta^{x} \mid A\right)=g_{A}^{x x}\left(f_{1}\left(\beta^{x} \mid A\right)\right)=g_{A}^{x x}\left(\beta^{x}\right)$. This proves that $\left(a^{x x}\right)^{\prime}=g_{A}^{x x}$ and consequently, $g_{A}^{x x} \in\left(A^{x x}\right)^{\prime}$. Similarly, we can show that $g_{B}^{x x} \in\left(B^{x x}\right)^{\prime}$. Hence $G^{x x}$ $=\left(A^{x x}\right)^{\prime} \times\left(B^{x x}\right)^{\prime}$ is a direct product of two closed normal subgroups $\left(A^{x x}\right)$, and $\left(B^{x x}\right)^{\prime}$. Define:

and

$$
h_{1}: A^{x x} \rightarrow\left(A^{x x}\right)^{\prime} \text { by } h_{1}\left(a^{x x}\right)=\left(a^{x x}\right)^{\prime}
$$

$$
h_{2}: B^{x x} \rightarrow\left(B^{x x}\right)^{\prime} \text { by } h_{2}\left(b^{x x}\right)=\left(b^{x x}\right)^{\prime} .
$$

It is not hard to see that $h_{1}$ and $h_{2}$ are topologically isomorphic onto mappings. Therefore, we have $G^{x x} \cong A^{x x} \times B^{x x}$.

Let $G_{d}^{x}, A_{a}^{x}$ and $B_{d}^{x}$ be the set of all unitary representations of $G, A$ and $B$ with the discrete topology, respectively. Let $\left(G_{d}^{x}\right)^{x},\left(A_{d}^{x}\right)^{x}$ and $\left(B_{d}^{x}\right)^{x}$ be the groups of all unitary mappings of $G_{d}^{x}, A_{d}^{x}$ and $B_{d}^{x}$ with the usual finite open topology, respectively. By the proof of Theorem 5, we have the following results.

COROLlaRY 1. Let $G$ be a direct product of two topological groups $A$ and $B$, $G=A \times B$. Then $\left(G_{d}^{x}\right)^{x}=\left(A_{d}^{x}\right)^{x} \times\left(B_{d}^{x}\right)^{x}$ is a direct product of $\left(A_{d}^{x}\right)^{x}$ and $\left(B_{d}^{x}\right)^{x}$.

Corollary 2. Let $G=A \times B$ be a direct product of two locally compact groups $A$ and $B$. Then

$$
\begin{aligned}
& 1 \rightarrow A \stackrel{i}{\rightarrow} G \stackrel{j}{\rightarrow} B \rightarrow 1, \\
& 1 \leftarrow A^{x^{i^{x}}} G^{x}{ }^{j^{x}} B^{x} \leftarrow 1,
\end{aligned}
$$

and

$$
1 \rightarrow A^{x x} \stackrel{i^{x x}}{\longrightarrow} G^{x x} \stackrel{j^{x x}}{\longrightarrow} B^{x x} \longrightarrow 1
$$

where $i, j, i^{x}, j^{x}$ are defined in Lemma 10 and $\left[i^{x x}\left(a^{x x}\right)\right]\left(g^{x}\right)=a^{x x}\left(i^{x}\left(g^{x}\right)\right)$ and $\left[j^{x x}\left(g^{x x}\right)\right]\left(b^{x}\right)=g^{x x}\left(j^{x}\left(b^{x}\right)\right), a^{x x} \in A^{x x}, g^{x} \in G, b^{x} \in B$, are exact and $i^{x x}$ and $j^{x x}$ are continuous and open with respect to their images.

COROLlaRY 3. Let $G$ be a locally compact maximally almost periodic group and $G=A \times B$ is a direct product of two closed normal subgroups $A$ and $B$. If both $A$ and $B$ have the unitary duality, then so has $G$.

Proof. Applying the five-lemma of algebraic topology (e.g. see p. 16 of [6]) to Corollary 2, we have the desired result. We notice that the five-lemma is known true for the case of abelian groups. However, it is still true for the case of nonabelian groups, if we read the proof of the lemma carefully. 
COROLlary 4. Let $G$ be a locally compact, connected maximally almost periodic group. Then $G$ has the unitary duality.

Proof. This is a direct consequence of Corollary 3, Theorem 3, Theorem 4, and a result of $H$. Freudenthal (see [7]) that says any such group $G$ can be decomposed into a direct product of two closed normal subgroups $A$ and $B$ such that $A$ is abelian and $B$ is compact.

6. Unitary duality on another class of groups. Let $G$ be a topological group. Let $R_{G}$ be the algebra of all finite linear combinations with complex coefficients $f(x)=\sum_{k=1}^{n} a_{k} d_{i j}^{k}(x)$ where $d_{i j}^{k}(x)$ is a coefficient of some $D^{k}(x)=\left(d_{i j}^{k}(x)\right) \in G^{x}$ with the usual uniform norm. We call $R_{G}$ the resprentative algebra of $G$ over the field of complex numbers.

LeMma 11. Let $G$ be a maximally almost periodic group. Then for every compact normal subgroup $K$ of $G$ we have the natural homomor phism $i^{*}: R_{G} \rightarrow R_{K}$ which is onto, where $i^{*}$ is induced by the inclusion $i: K \rightarrow G$ and the least ideal containing $\left(j^{*}\left(R_{G / K}\right), K\right)=\left\{f \mid f \in j^{*}\left(R_{G / K}\right)\right.$ and $f(k)=0$ for all $\left.k \in K\right\}$ is dense in $\left(R_{G}, K\right)$ where $j^{*}$ is induced by the natural projection $j: G \rightarrow G / K$.

Proof. We show $i^{*}: R_{G} \rightarrow R_{K}$ is onto. It is enough to show $i^{x}: G^{x} \rightarrow K^{x}$ is onto. It is clear that for each $g^{x} \in G^{x},\left(i^{x}\left(g^{x}\right)\right)^{-} \in i^{x}\left(G^{x}\right)$ and for each $g_{1}$ and $g_{2}$ in $G^{x}, i^{x}\left(g_{1}^{x} \times g_{2}^{x}\right)=i^{x}\left(g_{1}^{x}\right) \times i^{x}\left(g_{2}^{x}\right)$ and each irreducible part of $i^{x}\left(g_{1}^{x} \times g_{2}^{x}\right)$ over $K$ is in $1^{x}\left(G^{x}\right)$. By a well known theorem of Van Kampen (see [12]): "All continuous representations of any compact group $F$ can be obtained, by taking conjugates and decomposing direct products, from any collection of such representations, not all equal on any two distinct points of $F$.' Since $i^{x}\left(G^{x}\right)$ is such a subset of $K^{x}$, we have $i^{x}\left(G^{x}\right)=K^{x}$. This shows that $i^{*}$ is onto.

We show that the least ideal containing $\left(j^{*}\left(R_{G / K}\right), K\right)$ is dense in $\left(R_{G}, K\right)$. Let $\tilde{G}$ be the compact group attached to $G$ in the sense of Weil (see [19]) (the Bohr compactification of $G$, (e.g. see [2] and [3])) and a continuous homomorphism $f: G \rightarrow \tilde{G}$, with $(f(G))^{-}=\tilde{G}$, be defined naturally. Then $\left.K \cong f K\right) \cong \tilde{K}$ and $\tilde{G} / f(k)=(\tilde{G} / K)$. We may identify these groups, since $\left(R_{G}, K\right) \cong\left(R_{\tilde{G}},(K)\right.$ and $\left(j^{*}\left(R_{G / K}\right), K\right)=\left(j^{*}\left(R_{\tilde{G} / K}\right), K\right)$. Since $\tilde{G}$ is compact, by a usual technique in analysis it is not hard to see that the least ideal containing $\left(j^{*}\left(R_{G / K}\right), K\right)$ is dense in $\left(R_{\tilde{G}}, K\right)$.

Definition 5. Let $G$ be a maximally almost periodic, locally compact group. A representation of the algebra $R_{G}$ is an algebraic homomorphism $F$ of the algebra $R_{G}$ into the field of complex numbers such that $F(\bar{g})=(F(g))^{-}$for $g \in R_{G}$.

LEMMA 12. There is a one-to-one correspondence between the set of all representations of $R_{G}$ and the set $\left(\left(G^{x}\right)_{d}\right)^{x}$ of all unitary mappings of $\left(G^{x}\right)_{d}$, which is the set of all unitary representations of $G$ with the discrete topology.

Proof. For each representation $F$ of the algebra $R_{G}$, define $f(D)=F\left(d_{i j}\right)$ for $D=\left(d_{i j}\right) \in\left(G^{x}\right)_{d}$. It is easy to verify that $f \in\left(\left(G^{x}\right)_{d}\right)^{x}$. Using the fact that 
$\left(G^{x}\right)_{d} \cong\left(\tilde{G}^{x}\right)_{d}$, where $\tilde{G}$ is the compact group attached to $G$, we may complete the proof by imitating a method used by Chevalley (see [4, Proposition 2, p. 196]).

LEMMA 13. Every representation of $R_{G}$ is continuous with respect to the uniform norm of $R_{G}$.

Proof. Using the fact that $R_{G} \cong R_{\tilde{G}}$, where $\tilde{G}$ is the compact group attached to $G$, and the unitary duality on the compact group $\widetilde{G}$, we may prove this lemma by Lemma 12 and the unitary duality on compact groups (the Corollary 1 to Theorem $3)$.

Definition 6. A closed normal subgroup $B$ of a topological group $G$ is called extendible if the sequence

$$
1 \longrightarrow B^{x x} \stackrel{i^{x x}}{\rightarrow} G \stackrel{j^{x x}}{\longrightarrow}(G / B)^{x x} \longrightarrow 1
$$

is exact with $i^{x x}$ and $j^{x x}$ continuous, where $i^{x x}: B^{x x} \rightarrow G^{x x}$ defined by $i^{x x}(d)\left(g^{x}\right)$ $=\alpha\left(i^{x}\left(g^{x}\right)\right), \alpha \in B^{x x}$ and $g^{x} \in G^{x}$, where $i^{x}: G^{x} \rightarrow B^{x}$ defined by $i^{x}\left(g^{x}\right)(b)=g^{x}(i b)$, $g^{x} \in G^{x}, b \in B$.

It is trivial that every minimal almost periodic, closed normal subgroup of a minimal almost periodic group is extendible. By the Corollary 2 to Theorem 5 , the subgroup $A$ of $G=A \times B$, as direct product, is extendible. By Theorem 4, every closed subgroup of a locally compact abelian group is extendible.

Lemma 14. Let $G$ be a locally compact group. Let $A$ be an extendible subgroup of $G$, if $A$ and $G / A$ both have the unitary duality, then the mapping $\phi_{2}: G \rightarrow G^{x x}$, as defined in Lemma 8 , is continuously isomorphic onto.

Proof. Because of Lemma 8, it is sufficient to prove the mapping $\phi_{2}$ is one-toone and onto. Consider the following diagram:

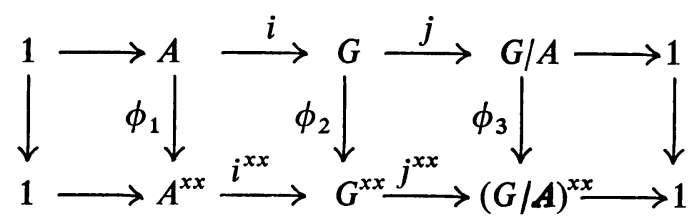

where $\phi_{1}, \phi_{2}, \phi_{3}$ are defined as in Lemma 8 . It is easy to verify that this diagram is commutative. By Lemma 8 , we know $\phi_{1}, \phi_{2}, \phi_{3}$ are continuously isomorphic into. Since $\phi_{1}$ and $\phi_{2}$ are also onto, by the well-known five-lemma (see the remark in the proof of Corollary 3 to Theorem 5), we know $\phi_{2}$ is a one-to-one and onto mapping.

LEMMA 15. Let $G$ be a maximally almost periodic, locally compact group. Let $A$ be a compact normal subgroup of $G$. If $G / A$ has the unitary duality, then $A$ is an extendible subgroup of $G$. 
Proof. Consider the commutative diagram in the proof of Lemma 14. We shall show that the sequence

$$
1 \longrightarrow A^{x x} \stackrel{i^{x x}}{\longrightarrow} G^{x x} \stackrel{j^{x x}}{\longrightarrow}(G / A)^{x x} \longrightarrow 1
$$

is exact. It is clear that $i^{x x}$ and $j^{x x}$ are continuous. The mappings $i$ and $\phi_{2}$ are isomorphic into and $\phi_{1}$ is isomorphic onto. This implies that $i^{x x}$ is isomorphic into. The mappings $j$ and $\phi_{3}$ are onto; this implies that $j^{x x}$ is onto. We shall show that $\operatorname{im}\left(i^{x x}\right)=\operatorname{ker}\left(j^{x x}\right)$. It is clear that $\operatorname{im}\left(i^{x x}\right) \subset \operatorname{ker}\left(j^{x x}\right)$. Let $F \in \operatorname{ker}\left(j^{x x}\right)=\left\{H \mid H \in G^{x x}\right.$, $\left.H\left(j^{x}(\alpha)\right)=1_{d(\alpha)} \alpha \in(G / A)^{x}\right\}$. From Lemma 7, we have $j^{x}(\alpha) \mid A=1_{d(\alpha)}$ for all $\alpha \in(G / A)^{x}$. By Lemma 12, we may consider $F$ as a representation of the representative algebra $R_{G}$. We shall show $F(\alpha)=0$ for each $\alpha \in\left(i^{*}\left(R_{G / A}\right), A\right)$. The subalgebra $j^{*}\left(R_{G / A}\right)$ is generated by all the coefficients (representative functions) in $j^{x}\left((G / A)^{x}\right)$. Since for each $\xi \in(G / A)^{x}$ and each $F \in \operatorname{ker}\left(j^{x x}\right), F\left(j^{x}(\xi)\right)=1_{d(\xi)}$ and $j^{x}(\xi) \mid A=1_{d(\xi)}$, every $\alpha \in\left(j^{*}\left(R_{G / A}\right), A\right)$ may be expressed as a polynomial $\alpha=\Sigma_{r_{i} \geqq 0 ; s_{j} \geqq 0} j_{r_{1} \ldots r_{m}} ; s_{1} \cdots s_{n} x_{1}^{r_{1}} \cdots x_{m}^{r} y_{1}^{s_{1}} \cdots y_{n}^{s_{n}}$ where $x_{i}$ and $y_{j}$ are coefficients of elements in $j^{x}\left((G / A)^{x}\right)$, respectively, such that $y_{j}$ are on the diagonals and $x_{i}$ are not. Consequentiy, $x_{i}\left|A=0, y_{j}\right| A=1, F\left(x_{i}\right)=0, F\left(y_{j}\right)=1$, and $\Sigma \alpha_{0} \cdots,,_{0} ; s_{1}, \cdots, s_{n}=0$. It follows that $F(\alpha)=0$, for all $\alpha \in\left(j^{*}\left(R_{G / A}\right), A\right)$. By Lemma 11, the least ideal containing $\left(j^{*}\left(R_{G / A}\right), A\right)$ is dense in $\left(R_{G}, i(A)\right)$. We have $F(d)=0$ for all $\alpha \in\left(R_{G}, i(A)\right)$ and from Lemma 11, we have $1^{*}: R_{G} \rightarrow R_{A}$ is onto, i.e., $R_{A} \cong R_{G} /\left(R_{G}, i(A)\right)$. Since $A$ is compact, by the Corollary 1 to Theorem 3 and Lemma 12, there exists an $a \in A$ such that $F(\xi)=\xi(a)$ for $\xi \in R_{G} /\left(R_{G}, A\right)$. For each $\xi \in\left(R_{G}, i(A)\right)$, we have $\xi(a)=0$ or $F(\xi)=0$. Consequently, we may say $F(\xi)$ $=\xi(i(A))$ for $\xi \in R_{G}$ or $F\left(g^{x}\right)=g^{x}(i a)$ for $g^{x} \in G^{x}$. We have $F\left(g^{x}\right)=i^{x} g^{x}(a)=$ $\phi_{1}(a)\left(i^{x} g^{x}\right)=\left(i^{x x}\left(\phi_{1}(a)\right)\right)\left(g^{x}\right)$. This shows that $i^{x x}\left(\phi_{1}(a)\right)=F$ and $\operatorname{im}\left(i^{x x}\right)=\operatorname{ker}\left(j^{x x}\right)$. Consequently, $A$ is extendible.

LFMMA 16. Let $G$ be a locally compact group with a $\sigma$-compact commutator subgroup. Let the group $G^{x x}$ be locally compact. If the homomorphism $\phi$ defined in Lemma 8 is onto then $\phi$ is open.

Proof. Let $M$ be the commutator subgroup of $G$. Choosing a compact neighborhood $U$ of the identity in $G$, the group $B=\bigcup_{n}\left(U M U^{-1}\right)^{n}$ is an open and closed normal subgroup of $G$. By Lemma $4,(G / B)_{1}^{x}$ is compact because $G / B$ is discrete. By Lemma 7 , we have $(G / B)_{1}^{x} \cong\left(G_{1}^{x}, B\right)$ and $\left(G_{1}^{x}, B\right)$ is compact subset of $G_{1}$. Let $B^{\prime}=\left\{f \mid f(\alpha)=1\right.$ for $\left.\alpha \in\left(G_{1}^{x}, B\right), f \in G^{x x}\right\}$. We shall show that $B^{\prime}$ is open. For any $\varepsilon, 1>\varepsilon>0$, let $\bigcup\left(e ;\left(G_{1}^{x}, B\right), \varepsilon\right)$ be a neighborhood of the identity $e$ in $G^{x x}$. Let $h \in \bigcup\left(e ;\left(G_{1}^{x}, B\right), \varepsilon\right)$. Then $h \mid G_{1}^{x} \in\left(G_{1}^{x}\right)_{1}^{x}$, which may be considered as the character group of $G_{1}^{x}$. Let $i:\left(G_{1}^{x}, B\right) \rightarrow G_{1}^{x}$ be the inclusion mapping. Then $i_{1}^{x}:\left(G_{1}^{x}\right)_{1}^{x} \rightarrow\left(\left(G_{1}^{x}, B\right)\right)_{1}^{x}$ where $\left(G_{1}^{x}, B\right)_{1}^{x}$, as the character group of compact abelian group $\left(G_{1}^{x}, B\right)$, is discrete. For each $\alpha \in\left(G_{1}^{x}, B\right)_{1}^{x}, h(\alpha)=\left(h \mid G_{1}^{x}\right)(\alpha)$. Any neighborhood 
$W\left(e ;\left(G_{1}^{x}, B\right), \varepsilon\right)$ of the identity $e$ in $\left(G_{1}^{x}, B\right)_{1}^{x}$ with $0<\varepsilon<1$, from Lemma 6 , contains the identity $e$ only. Hence $\left(i_{1}^{x}\left(h \mid G_{1}^{x}\right)\right)(\alpha)=1$ for $\alpha \in\left(G_{1}^{x}, B\right)$ or

$$
\left(i_{1}^{x}\left(h \mid G_{1}^{x}\right)\right)(\alpha)=h \mid G_{1}^{x}(i \alpha)=h(\alpha)=1 .
$$

This shows that $h \in B^{\prime}$ and $\bigcup\left(e ;\left(G_{1}^{x}, B\right), \varepsilon \subset B^{\prime}\right.$. Consequently, $B^{\prime}$ is an open subgroup.

We shall show that $\phi(B)=B^{\prime}$. It is clear that $\phi(B) \subset B^{\prime}$. Let $a \in B^{\prime} \mid \phi(B)$. Since $\phi$ is onto, there exists an $x \in G$ such that $\phi(x)=a$ and $x B \in G^{\prime} \mid B$. From the fact that $G / B$ is a locally compact abelian group, $(G / B)_{1}^{x}=\left(G_{1}^{x}, B\right)$, and $x B$ is not the identity in $G / B$, there exists $\alpha \in\left(G_{1}^{x}, B\right)$ such that $\alpha(x) \neq 1$. This shows $\phi(x)(\alpha)$ $=a(\alpha) \neq 1$ and $a \notin B^{\prime}$. A contradiction! Since $B$ and $B^{\prime}$ are locally compact and $B$ is $\sigma$-compact, by a well-known theorem $\phi$ is open from $B$ onto $B^{\prime}$. From the fact that $B^{\prime}$ is open, the lemma is proved.

THEOREM 6. Let $G$ be a maximally almost periodic, locally compact group with a $\sigma$-compact commutator subgroup. Let $A$ be a compact normal subgroup of $G$. If $G / A$ has the unitary duality, so does $G$.

Proof. By Lemma 15 and Lemma 14, we know the mapping $\phi_{2}: G \rightarrow G^{x \boldsymbol{x}}$, as defined in Lemma 8 , is continuously isomorphic onto. It is sufficient to prove that $\phi_{2}$ is open. By Lemma 16 , it is enough to prove that $G^{x x}$ is locally compact. Consider the commutative diagram in the proof of Lemma 14. From the fact that $\phi_{2}$ is one-to-one and $j$ and $\phi_{3}$ are open, it follows that $j^{x x}$ is open. From the exact sequence

$$
1 \longrightarrow A^{x x} \stackrel{i^{x x}}{\longrightarrow} G^{x x} \stackrel{j^{x x}}{\longrightarrow}(G / A)^{x x} \longrightarrow 1
$$

with $i^{x x}$ and $j^{x x}$ open, continuous and $A^{x x}$ and $(G / A)^{x x}$ are locally compact, we have $G^{x x}$ is locally compact. The theorem is proved.

COROLlaRy 1. Let $G$ be a maximally almost periodic, locally compact group with a compact commutator subgroup. Then $G$ has the unitary duality.

The above result is similar to a result of Takahashi (see [17]). In fact, the difference is that in his paper he used two different topologies: for $G^{x \boldsymbol{x}}$ he used the usual compact open topology and for $G^{x}$ he did not. In this paper, we use the compact open topology for both. However, we reproduced some of his techniques in the proofs of Lemma 15 and Lemma 16.

7. The compact group attached to a topological group. Let $G$ be a topological group. We denote $\tilde{G}$ the compact group attached to $G$. (See [13] or [19].) Let $f: G \rightarrow H$ be a continuous homomorphism from a topological group $G$ into a topological group $H$. We denote the induced, continuous homomorphism by $\tilde{f}$ 
and $\tilde{G}$ into $\tilde{H}$. The following theorem suggests a new way to construct the compact group attached with a topological group other than the known methods (see [13] and [19]).

THEOREM 7. Let $G$ be a topological group. Then $\left(G_{a}^{x}\right)^{x} \cong \tilde{G}$.

Proof. Since the sets $G_{d}^{x}$ and $(\widetilde{G})_{d}^{x}$ are same (see [19]), we have $\left(G_{d}^{x}\right)^{x} \cong\left((\widetilde{G})_{d}^{x}\right)^{x}$. By the Corollary 1 to Theorem 3 , we have $\left((\widetilde{G})_{d}^{x}\right)^{x} \cong \widetilde{G}$, since $\widetilde{G}$ is compact.

COROLlaRY 1. Let $G$ be a topological, abelian group. Then the character group with the finite open topology of the character group of $G$ with the discrete topology is the compact group associated with $G$, i.e., $\left(\left(G_{1}^{x}\right)_{d}\right)_{1}^{x} \cong \tilde{G}$.

Proof. This Corollary is a direct consequence of Theorem 4, Corollary 1 to Theorem 3 and Theorem 7.

In [2] and [3], Professor Kakutani established the same results for the case that $G$ is either the additive group of all integers with the discrete topology or the additive group of all real numbers with the usual topology.

Corollary 2. Let

$$
\cdots \longrightarrow A_{1} \stackrel{f_{1}}{\longrightarrow} A_{2} \stackrel{f_{2}}{\longrightarrow} A_{3} \stackrel{f_{3}}{\longrightarrow} A_{4} \longrightarrow \cdots
$$

be an exact sequence of locally compact abelian groups such that $f_{1}, f_{2}, f_{3}$, are continuous and open with respect to their images. Then

$$
\cdots \longrightarrow \tilde{A}_{1} \stackrel{\tilde{f}}{\longrightarrow} \tilde{A}_{2} \stackrel{\tilde{f}_{2}}{\longrightarrow} \tilde{A}_{3} \stackrel{\tilde{f}_{3}}{\longrightarrow} \tilde{A}_{4} \longrightarrow \cdots
$$

is exact.

Corollary 3. Let $G=A \times B$ be a direct product of two topological groups. Then $\tilde{G}=\tilde{A} \times \tilde{B}$.

Proof. It is a direct consequence of the Corollary 1 to Theorem 5 and of Theorem 7.

COROLlaRY 4. The image of the group $G$ is one-to-one into $\tilde{G}$ by $f$ if and only if $G$ is maximally almost periodic, where $f$ is the natural continuous homomorphism from $G$ into $\tilde{G}$.

THEOREM 8. Let $G$ be a locally compact group. Then $\left(\tilde{G}^{x x}\right)=\tilde{G}$.

Proof. Consider the following commutative diagram:

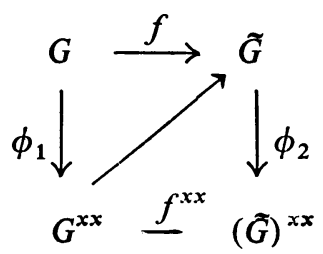


where $f$ is the natural, continuous homomorphism from $G$ into $\tilde{G}, f^{x x}$ is induced by $f$, and $\phi_{1}$ and $\phi_{2}$ are defined in Lemma 8. From Theorem 3, we know $\phi_{2}$ is a topological isomorphism. Consequently, there is a continuous homomorphism $h$ from $G^{x x}$ into $\tilde{G}$ such that $h \circ \phi_{1}=f$. Both the image of $f$ and the image of $h$ are dense in $G$ and $\widetilde{G}$ respectively.

Now the statement follows obviously from the characterization of the compact group $\tilde{G}$ attached to $G$ by Weil's procedure that, if the natural homomorphism $f: G \rightarrow \widetilde{G}$ can be written as $f=h \circ \phi$ where $\phi: G \rightarrow G^{\prime}$ and $h: G^{\prime} \rightarrow \widetilde{G}$ are continuous homomorphisms and $G^{\prime}$ is a topological group, then $\tilde{G}^{\prime} \cong \tilde{G}$.

If $G$ is maximally almost periodic, locally compact, with a compact commutator subgroup, then by the Corollary 1 to Theorem 6 , we know the homomorphism $\phi$ above is topologically isomorphic onto. The author does not know whether the condition that the commutator subgroup be compact is necessary for the conclusion that $\phi$ is a topological isomorphism.

The author cannot answer the question even for the case of the free group with two generators (with the discrete topology).

The author wishes to take this opportunity to express his indebtedness to Morikumi Goto for his encouragement.

\section{REFERENCES}

1. El-Ichi Abe, Dualité de Tannaka des groupes algébriques, Tôhoku Math. J. 12 (1960), 327-332.

2. H. Anzai and S. Kakutani, Bohr compactifications of a locally compact Abelian group. I, Proc. Imp. Acad. Tokyo 19 (1943), 476-480.

3. - Bohr compactifications of a locally compact Abelian group. II, Proc. Imp. Acad. Tokyo 19 (1943), 533-539.

4. C. Chevalley, Theory of Lie groups, Princeton Univ. Press, Princeton, New Jersey, 1946.

5. P. Cartier, Dualité de Tannaka des groupes et algebre de Lie, C. R. Acad. Sci. Paris 242 (1956), 322-324.

6. S. Eilenberg and N. Steenrod, Foundations of algebraic topology, Princeton Univ. Press, Princeton, New Jersey, 1952.

7. H. Freudenthal, Topologische Gruppen mit genugend vielen fastperiodischen Funktionen, Ann. of Math. (2) 37 (1936), 57-75.

8. M. Goto, Note on a topology of a dual space, Proc. Amer. Math. Soc. 12 (1961), 41-46.

9. G. Hochschild and G. Mostow, Representations and representative functions of Lie groups, Ann. of Math. (2) 66 (1957), 495-542.

10. N. Iwahori and N. Iwahori, An extension of Tannaka duality theorem for homogeneous spaces, Sci. Papers Coll. Gen. Ed. Univ. Tokyo 8 (1958), 115-121.

11. G. I. Kac, Generalization of the group principle of duality, Dokl. Acad. Nauk SSSR 138 (1961), 275-278 = Soviet Math. Dokl. 2 (1961), 581-584.

12. Van Kampen, Almost periodic functions and compact groups, Ann. of Math. (2) 37 (1936), 78-91.

13. L. H. Loomis, An introduction to abstract harmonic analysis, Van Nostrand, New York, 1953. 
14. J. von Neumann, Almost periodic functions in a group. I, Trans. Amer. Math. Soc. 36 (1934), 445-492.

15. L. Pontrjagin, Topological groups, Princeton Univ. Press, Princeton, New Jersey, 1939.

16. W. F. Stinespring, Integration theorems for gages and duality for unimodular groups, Trans. Amer. Math. Soc. 90 (1959), 15-56.

17. S. Takahashi, $A$ duality theorem for representable locally compact groups with compact commutation subgroup, Tôhoku Math. J. 5 (1953), 115-121.

18. T. Tannaka, Über den dualitotssatz der nicht kommutativen topologischen gruppen, Tôhoku Math. J. 45 (1938), 1-12.

19. A. Weil, L'intégration dans les groupes topologiques et ses applications, Actualités Sci. Ind. No. 869, Hermann, Paris, 1960.

\author{
UNiversity of British COLUMBIA, \\ Vancouver, British Columbia, Canada \\ RESEARCh INSTITUTE, UniVERSITY OF Alabama, \\ Huntsville, Alabama \\ UNIVERSITY OF MARYLAND, \\ College Park, Maryland
}

